Corrigenda et Errata OTE 30/1 (2017): $205 \quad 205$

\title{
Corrigenda et Errata
}

The Doi number of the editorial in OTE 29 (3) 2016 has been rendered incorrectly. It should read http://dx.doi.org/10.17159/2312-3621/2016/v29n3a2 